\title{
Adaptation Asymmetry in Manual Tracking
}

\section{Erwin R. Boer $\dagger \&$ Robert V. Kenyon $\ddagger$}

\author{
$\dagger$ Cambridge Basic Research, Nissan R\&D Inc. \\ 4 Cambridge Center, Cambridge, MA 02142 \\ Tel. 617-374-9663, Email: erwin@pathfinder.cbr.com \\ $\ddagger$ University of Illinois at Chicago, Dept. EECS (M/C 154), Rm. 1120, SEO Bldg. \\ 851 S. Morgan, Chicago, IL 60607-7053 \\ Tel. 312-996-0450, Email: kenyon@eecs.uic.edu
}

\begin{abstract}
A theory of manual adaptive control requires an understanding of the mechanisms by which human operators alter their control strategies in response to changes in control conditions. Here we show that significant asymmetries in adaptation occur in a manual tracking task when well-trained subjects are exposed to situations in which track preview, lag of the controlled system, or track bandwidth gradually change. We analyzed the experimental data using our recently developed recursive system identification algorithm which not only estimates non-stationary linear model coefficients but also time varying delay time. We show that daptation strongly depends on whether the task becomes easier or more difficult as a result of changing conditions. Subjects were not always inclined to improve their strategy when tracking difficulty gradually decreases as a result of changing conditions. When the task gradually became more difficult, subjects seemed predisposed to maintain their current strategy rather than switching to a more effective strategy particlularly when switching required an increased work load or attentional demand. The observed adaptation asymmetries indicate that tracking performance can not be predicted based on momentary task conditions alone. To establish a model of adaptation mechanisms and its driving forces, the recent history of changes in tracking conditions needs to be considered. We attribute the observed asymmetries to a performance judgment process which triggers adaptation when perceived performance falls outside a subjective range that depends strongly on the recent history of tracking conditions.
\end{abstract}

\section{Introduction}

In his 1969 paper Young expressed the challenge for future work in manual control: "Only by pressing the development of a theory of manual adaptive control for the unlikely and unexpected failure will we keep the theory of manual control relevant to the needs of the times" [9]. Focus has since been directed primarily to the human as a supervisory controller [7]. One reason for the evidential abandonment of adaptive manual control was the lack of a technique for dynamic mea- surement of the adaptive processes that take place in a human operator when the control task changes either abruptly or gradually.

Models for human operator response to sudden changes in control conditions are much better understood than those to gradual changes and were researched extensively during the sixties $[3,6]$. These models generally consist of a detection, an identification and a modification stage. Adaptation is often reduced to switching from one well learned control mode to another. An overview of the experiments, theories and analyses used to characterize human adaptation is given in [8].

The need for better measurement tools was however never addressed satisfactorily. The main problem lies in the difficulty to recursively identify time varying delay time. Identification of delay time is crucial since it can change considerably during manual adaptation [5]. Recently we have been able to simultaneously identify the changing linear coefficients of a system as well as its changing delay time [2]. In this paper, we use this identifier to characterize human operator adaptation to gradual changes in a manual tracking task. Our focus is directed to the effect of changing preview, track bandwidth and control plant dynamics on human operator behavior and show that adaptation depends asymmetrically on the direction of the changing conditions. We hypothesize that perceived performance and workload play a significant role in mediating human adaptation to different varying control conditions. Our long term goal is to use the recursive identifier to develop a theory of manual adaptive control.

\section{Experimental Design}

A detailed description of the experimental design can be found in [1]. A brief description follows.

Subjects sat $75 \mathrm{~cm}$ away from a 19 inch graphics monitor and superimposed a cross over a reference track using a joystick (Fig. 2). The springless joystick (Measurement Systems Inc. Model 521) had adjustable kinematic friction and no equilibrium point. This friction remained constant for all 
deflection angles and was set to satisfy the subjective preference across all subjects. Static friction was negligible. Stick data, with defection angles ranging between -20 and +20 degrees, was sampled at $60 \mathrm{~Hz}$ using a 12 bit A/D converter. The visual scene was updated at the same rate.

Trials were characterized by three experimental parameters: preview length $(\mathrm{PV})$, reference track bandwidth (BW), and control plant cutoff frequency (COF). Trials with fixed settings as well as trials in which one or two of the experimental parameters changed over time were presented to the subjects.

The reference track was constructed based on a sum of 16 sinusoids with frequencies between 0.1 and $1.0 \mathrm{~Hz}$, equal amplitudes, and random phases. The resulting signal was normalized to an RMS of $4.3 \mathrm{~cm}$ to create the reference track as illustrated by the solid line in Fig. 1. To create a track with time varying bandwidth, this signal was subsampled at appropriate intervals.
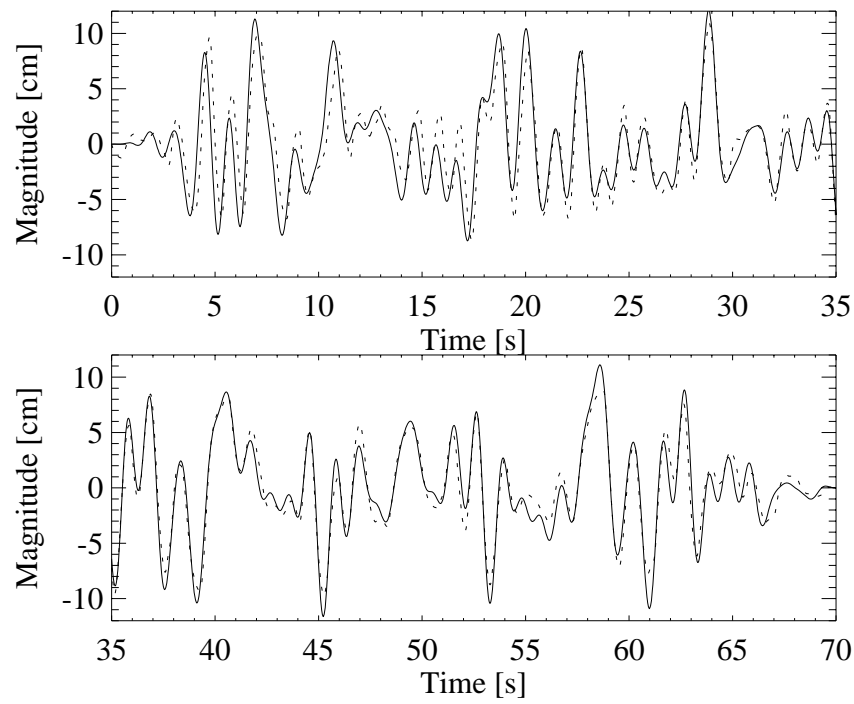

Figure 1: Subject PB's raw data during an increasing preview trial. Track bandwidth was $1.0 \mathrm{~Hz}$ and the control plant's cutoff frequency $0.75 \mathrm{~Hz}$. The solid line represents the reference track and the dotted line the plant output.

The gain of the first order stable control plant was adjusted to assure unit gain at $0.5 \mathrm{~Hz}$ regardless of the pole placement. This reduced the otherwise large fluctuations in perceived stick sensitivity when the control plant cutoff frequency changes.

All six student subjects (two female and four male) participated in three 45-min training sessions followed by six 45min experimental sessions. The training sessions familiarized them with all the possible conditions they would encounter in the experimental sessions. A maximum of three session per day was adopted with at least 30 minutes between sessions. Each subject performed a total of 80 trials, lasting $70 \mathrm{~s}$ each, during the three training session and 167 trials during the six experiment sessions.
The different preview conditions are described in the caption of Fig. 2.

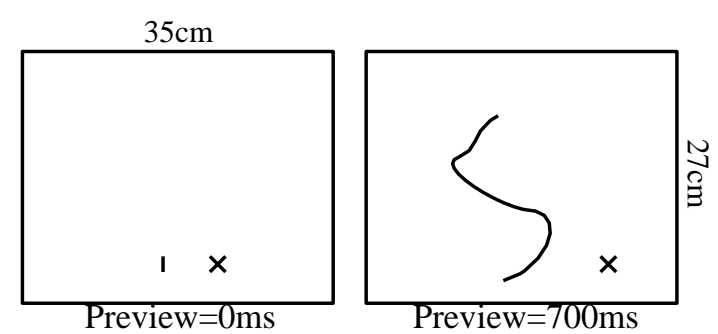

Figure 2: Experimental visual scene as presented on 19inch graphics monitor. Left panel shows the zero preview case in which no directional information was available. Right panel shows approximately how far the reference signal extended into the future (toward top of screen) with $700 \mathrm{~ms}$ preview. The top of the screen corresponded to $1.0 \mathrm{~s}$ preview, but this preview amount was never used.

Trial conditions were picked randomly without replacement from the following set of experimental parameter settings. The static conditions consisted of all permutations on $(\mathrm{PV}=0,167,700 ; \mathrm{BW}=0.5,1.0 ; \mathrm{COF}=0.75,2.5)$. The time varying trials consisted of permutations on $(\mathrm{PV}=0-700$; $\mathrm{BW}=0.5,1.0 ; \mathrm{COF}=0.75,2.5)$, on $(\mathrm{PV}=0,167,700 ; \mathrm{BW}=0.25$ $1.0 ; \quad \mathrm{COF}=0.75,2.5)$, on $(\mathrm{PV}=0,167,700 ; \quad \mathrm{BW}=0.5,1.0$; $\mathrm{COF}=0.5-2.5)$, and on all permutation in which two experimental parameter varied. $\mathrm{PV}=0-700$ indicates that preview changed from 0 to $700 \mathrm{~ms}$ or from 700 to $0 \mathrm{~ms}$. Every static conditions was run four times and every time varying conditions twice. For example, subjects experienced increasing preview twice and decreasing preview twice for the same BW and COF settings. In this paper we only report on a subset of the trials in which one experimental parameter changed.

Experimental parameters remained constant during the first and last 15 s of the time varying trials and changed linearly between the specified values when time ranges from 15 to $55 \mathrm{~s}$. Their values are indicated along the $\mathrm{x}$-axis in Figs. 3 through 5 .

\section{Analyses}

Man-Machine System Model Estimating human operator coefficients requires knowledge about the input signal they use. In compensatory and pursuit tracking the input signal is well defined. However, during preview tracking, operators have flexibility in shifting their input signal back and forth in time by changing the point at which the fixate in the preview. Without monitoring eye position, exact knowledge about the input signal can only be inferred. To circumvent this problem, we decided to identify the entire closed loop system rather than just the human operator. This provides information regarding the operator's ability to turn the entire system into a unit-gain, zero-delay tracking system. To assess this, the model of the MMS or man-machine system (i.e. human-plus-control-plant) was assumed to be accu- 
rately characterized by the following discrete time ARMAX $(1,1,2)$ model

$$
\begin{aligned}
y_{n}= & a_{1} y_{n-1}+b_{0} r_{n+d_{n}}+b_{1} r_{n-1+d_{n}}+ \\
& v_{n}+c_{1} v_{n-1}+c_{2} v_{n-2}
\end{aligned}
$$

where $d_{n}$ is the time varying fractional delay time. This model is most appropriate if the majority of time is spend in open loop mode; during pursuit (and preview) tracking humans operate in open loop control mode for extended periods of time with only intermittent transitions to compensatory or error correcting control mode [4]. Until we have established a clear understanding of the mechanisms responsible for switching between control modes, we assume that the effect of infrequent error corrections is small and can be characterized by colored noise as indicated in proposed model.

Recursive Model Identification Simultaneous estimation of time varying delay time and linear model coefficients was performed using the recursive identification technique detailed in [2]. This technique employs the Extended Kalman Filter to recursively estimate fractional delay time which is then used to resample the input signal using bilinear interpolation. The resulting locally time-shifted input signal is subsequently used to estimate the linear model coefficients for the current time step using the normal Kalman Filter $^{1}$. The identifier was run in non-causal or smoothing mode to eliminate the estimation lag that would have been introduced otherwise. This effectively eradicates the need to question whether the observed asymmetry in adaptation is true or an artifact of the identifier.

Performance Measure To test our hypothesis that the perceived changes in performance, resulting from changing tracking task conditions, plays a role in mediating adaptation, the following algorithm was developed to compute a recursive measure of tracking error. This algorithm sorts the squared tracking errors in a 10 s window centered on the time step of interest and returns the squared tracking error value that is larger than $85 \%$ of the values in this window ${ }^{2}$. The results are shown in the bottom panels of Figs. 3 through 5 .

\section{Results}

Raw data of an increasing preview trial is shown in Fig. 1. The plant output is draw as the dotted line. It shows the improvement in tracking as preview increases as indicated by the decrease in the number of large over and under shoots

\footnotetext{
${ }^{1}$ The a-priori noise covariance $R$ was set to $5.0 e-2$, the variance in delay time $Q_{d}$ to $5.0 e-2$ and the variance in the linear coefficients $Q_{p}$ to $5.0 e-5$. Note that only the ratio between $Q_{d}, Q_{p}$ and $R$ affects the identifier. The results shown in Figs. 3 through 5 were obtained using these settings. Changes in these coefficients on the order of $50 \%$ did not affect the results in a significant manner.

${ }^{2}$ Using a forward/backward running average with forgetting factor required such a long effective averaging window to reduce large fluctuations that it lost its time specificity. This motivated our choice of the 85 percentile measure.
}

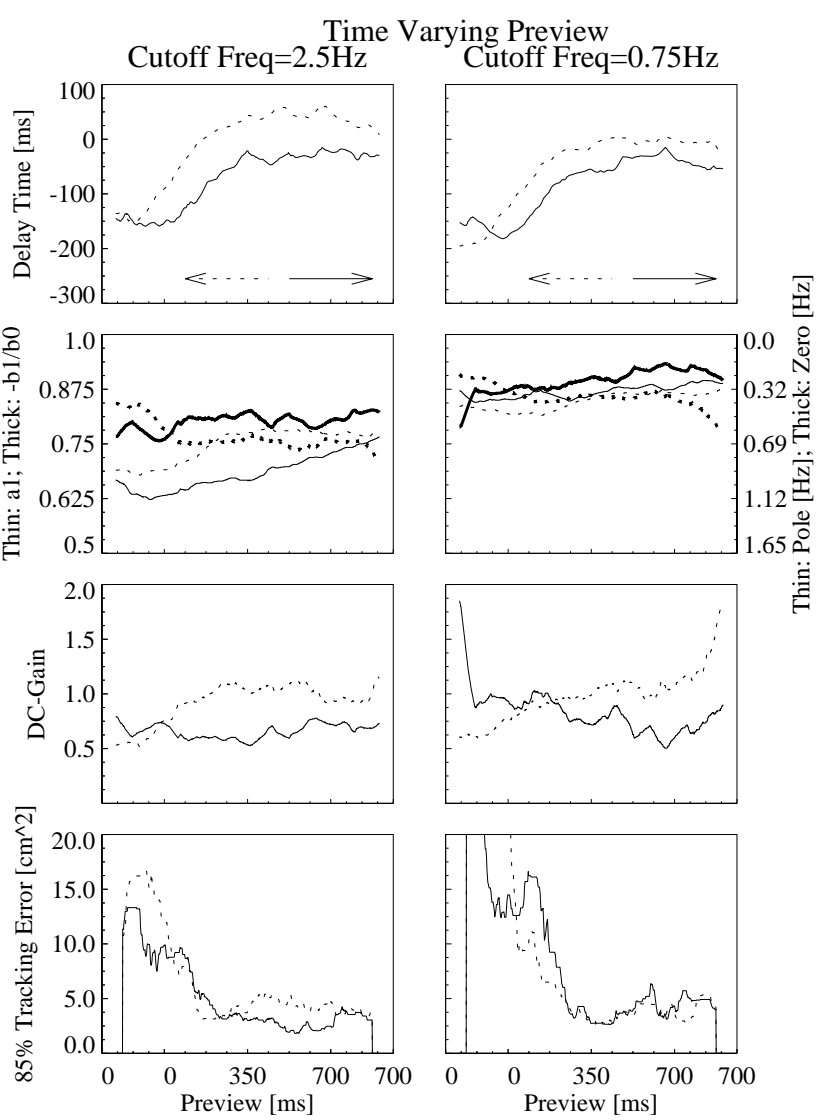

Figure 3: Human operator adaptation to changing preview. Two conditions are compared; left (right) column shows the effect at a $2.5 \mathrm{~Hz}(0.75 \mathrm{~Hz})$ control plant cutoff frequency. The track bandwidth was fixed at $1.0 \mathrm{~Hz}$ in both cases. Solid (dotted) lines indicate an increase (decrease) in preview according to the scale along the $x$-axis. See text for further details.

as well as a decrease in the time shift between reference track and plant output.

The effects of changes in the experimental parameters (preview, track bandwidth and control plant cutoff frequency) on the overall identified system are shown in Figs. 3 through 5. The experimental conditions are explained in the figure captions. Median results of the four most motivated subjects (two males and two females) are shown; they reflect the typical adaptation asymmetries observed in all subjects. Primary focus in this paper is directed to the effect of changing control conditions on delay time adaptation. Changes in pole-zero placement and dc-gain are addressed only briefly. Presentation of all the results is beyond the scope of this paper and will be topic of a forthcoming journal paper.

In each figure, the top panel shows delay time, the next pole and zero placement, the third row shows dc-gain, and the bottom panels the 85 percentile squared tracking error It is important to note that the solid (dotted) lines represent 
trials in which the task difficulty decreased (increased). To allows for direct comparison of model coefficients under identical experimental conditions, trials with increasing task difficulty (dotted lines) are plotted in reverse time. This means that time runs from right to left for the dotted lines as indicated by the dotted arrow in the top panels. For example, in the top panel of Fig. 3 the solid arrow, which corresponds to the data represented in solid lines, indicates that time runs from left to right and that preview increases from 0 to $700 \mathrm{~ms}$. Similarly, the dotted arrow in the top panel of Figure 4 indicates that time runs from right to left and that track bandwidth increases from 0.2 to $1.0 \mathrm{~Hz}$. Even though data are plotted against time, values of the experimental variable at $0,15,35,55$ and 70 s are indicated along the $\mathrm{x}$-axis. Note that the experimental parameters remained constant for first and last $15 \mathrm{~s}$ of every trial as noted by the lack of changes in $\mathrm{x}$-axis values in this region.

The operator was able to null the total MMS delay time when preview reached about $300 \mathrm{~ms}$ (Fig. 3). This coincides with the point at which tracking error asymptotes. Decreasing preview (dotted lines) results in a slightly shorter delay time than observed during increasing preview with time. Dcgains are highest at the beginning of a trial particularly in case of a sluggish plant $(0.75 \mathrm{~Hz})$. The pole of the system during increasing preview and a responsive plant, lies at a higher frequency than observed during decreasing preview. This means that the total phase lag at frequencies greater that about $0.5 \mathrm{~Hz}$ may be similar for both conditions. Overall, the effects of increasing and decreasing preview with time follow a similar pattern and do not depend strongly on plant dynamics.

Adaptation to increasing track bandwidth with time (Fig. 4 , dotted lines) differs substantially from those observed during decreasing bandwidth. Subjects do not reduce delay time when bandwidth decreases with time even though they have exhibited shorter delay times for the same track bandwidth in decreasing bandwidth trials (dotted line above solid line at low bandwidth). Preview diminishes this asymmetry. During increasing bandwidth trials, preview also increases the bandwidth at which a constant delay time could be maintained (the dotted line bends down at higher frequencies as preview increases). This bend also signifies the point where tracking error starts to increase significantly.

In response to time varying track bandwidth, little asymmetry is observed in pole-zero placement. Similar to the observation made in response to time varying preview, the dc-gain seems highest during trial onset and then gradually decrease.

Changes in the MMS's dynamical are also observed in response to time varying control plant dynamics (Fig. 5). Delay time as well as the MMS's pole-zero placement show asymmetries. Recall that the control plant is most responsive at the $2.5 \mathrm{~Hz}$ cutoff frequency and is perceived as very sluggish when the cutoff frequency is around $0.5 \mathrm{~Hz}$. For subjects to equalize the lag introduced by the sluggish plant, they need to use lead equalization or prediction.

Delay time remains relatively constant when the plant

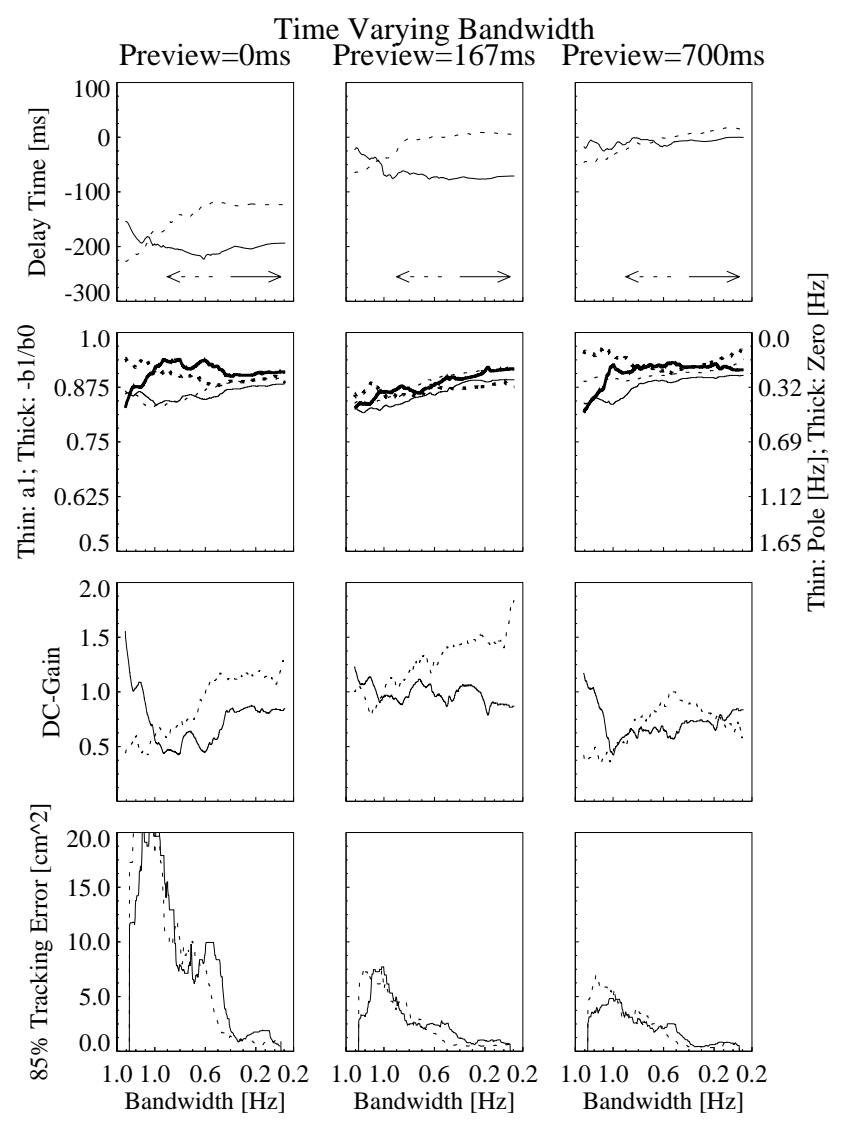

Figure 4: Human operator adaptation to changing track bandwidth. Three conditions are compared; left (middle,right) column shows the effect at a $0 \mathrm{~ms}$ (16\% $\mathrm{ms}, 700 \mathrm{~ms})$ preview. The control plant cutoff frequency remained at $0.75 \mathrm{~Hz}$ in all these trials. Changes in track bandwidth are shown along the $x$-axis. The information displayed in the different rows equals those in Fig. 3.

becomes more responsive (solid lines). Conversely, as the plant becomes more sluggish with time, a clear trend towards longer delay times is observed (dotted lines). These two lines cross at a cutoff frequency that increases with preview.

Since the dynamics of the system are actively altered as part of the experiment, changes in the MMS pole placement (thin lines) might be expected. In response to these changes in plant dynamics subjects modified their lead equalization as indicated by the sloping thick lines. Lead equalization is characterized by the fact that the zero's cutoff frequency falls at a lower frequency than the pole's cutoff frequency (thick line above thin line) thus effectively increasing the bandwidth of the system. Dc-gain and pole-zero placement also show asymmetries. When the plant starts out sluggish, subjects focus primarily on low frequencies in the reference track and adopt rate control as indicated by the high dc-gain and the fact that zero placement falls at a higher frequency 
than the pole placement (thin solid line above thick solid line when cutoff frequency is low). As the system becomes more responsive, the dc-gain drops significantly and the pole and zero placement lines cross. This high dc-gain and swapping of pole and zero placement is not observed when the system starts out responsive and reaches the sluggish regime.

As expected, performance improves significantly when preview is available. Here we clearly see the effect of asymmetries in model parameters on performance. At low cutoff frequencies, the dotted lines indicate worse performance. The main difference in model parameters is the lower dc-gain and longer delay time at the end of decreasing cutoff frequency trials. Similar correlations between asymmetries in model parameters on performance are observed for varying preview and track bandwidth.

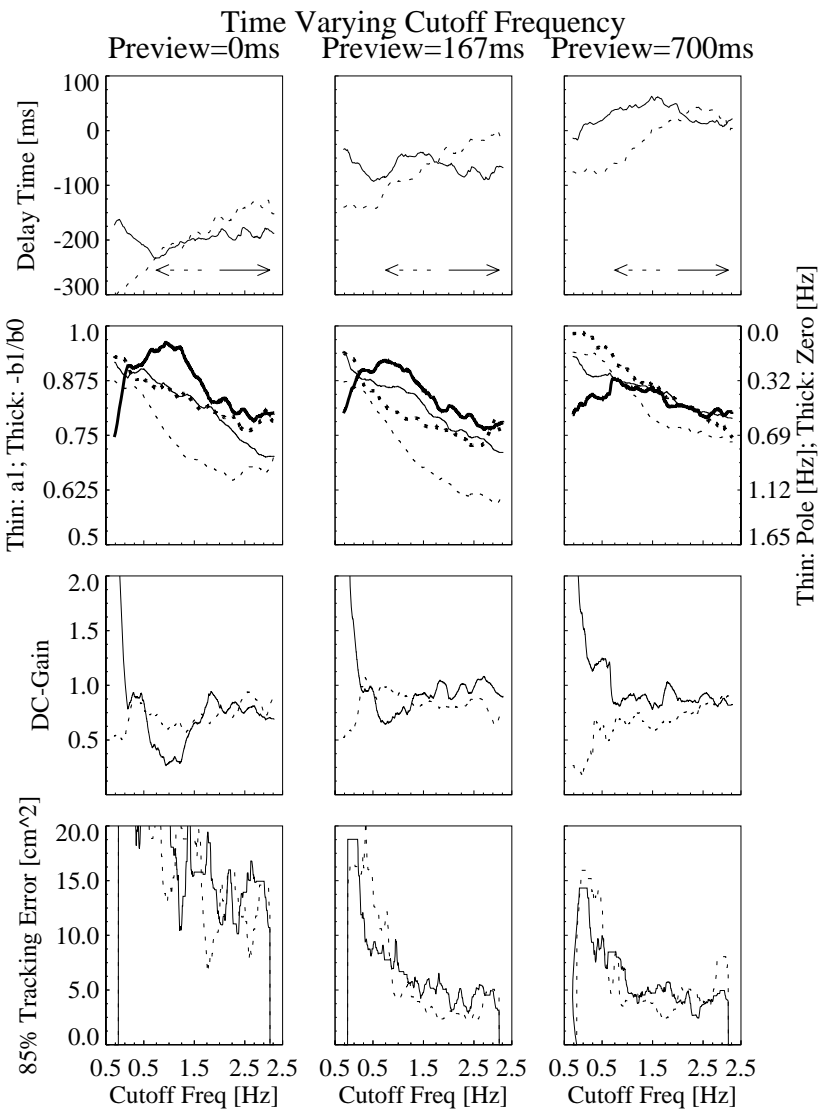

Figure 5: Human operator adaptation to changes in the first order control plant's cutoff frequency. Three conditions are compared; left (middle,right) column shows the effect at a Oms $(167 \mathrm{~ms}$,700ms) preview. The track bandwidth remained at $1.0 \mathrm{~Hz}$ in all these trials. Changes in plant cutoff frequency are shown along the $x$-axis. The information displayed in the different rows equals those in Fig. 3.

\section{Discussion}

Four types of control adaptation can be distinguished: input adaptation and prediction, task adaptation, controlled element adaptation and programmed adaptation [9]. In adaptive manual control, humans use their ability to select the most informative visual cues, establish a mental or internal model of the control plant and reference track characteristics, and establish a performance criterion that integrates task requirements and human processing and control limitation.

Gradual changes in control conditions are either perceived directly or need to be inferred from changes between the observed and expected effect of control actions. Conditions under which human operators perceive these gradual changes and under what conditions they will adapt or optimize their control strategy is topic of this discussion.

Adaptation to preview largely follows expectation in that preview provides the information human operators need to null the effect of their own inherent delay time as well as equalize the dynamical lags introduced by the plant and their own neuromusclar system. Since bandwidth can be perceived directly at long preview, the lack of a significant adaptation asymmetry to increasing and decreasing track bandwidth at $700 \mathrm{~ms}$ preview might be expected. Adaptation to changes in control conditions that require inference for detection, follows an asymmetrical and less predictable pattern. We suggest that subjective evaluation of perceived performance is one component responsible for the observed asymmetry in adaptation.

Preview is directly perceived while track bandwidth and plant dynamics need to be inferred. Given that perception is immediate but that inference takes time, if a single mechanism was involved in mediating the changes in overall system characteristics to increasing and decreasing track bandwidth conditions, then a hysteresis in the response curves would be expected. However, our results show an asymmetry rather than a hysteresis between these response curve indicating that different mechanisms may be responsible for each.

When a tracking task started out difficult (high bandwidth or low control plant cutoff frequency) and became gradually easier, subjects maintained their initial delay time until the end of the 70s trial even though they have demonstrated the ability to track with lower delay times under identical conditions. One explanation is that subjects realized that the task difficulty automatically decreased and did not see the need to improve performance even further. It is important to note that the performance measure at the bottom row in each figure may not be representative of the one subjects used internally. The displayed measure is also rather insensitive because of the 10 s sliding window used to compute the 85 percentile tracking error. From these results one may conclude that the subjects did not always optimize to their ability. Whether a fluid subjective tradeoff between performance and effort causes these discrepancies remains to be determined.

Subjective performance evaluation can explain only part of the observed adaptation asymmetry. A second process becomes apparent most clearly in the time varying cutoff 
frequency trials. When the system starts out responsive and then gradually turns sluggish, subjects do not lower their delay time nor do they increase their gain and switch to rate control. One possible explanation is that their perceived benefit to switch control strategy and adopt the more appropriate rate control does not outweigh the incurred cost of switching to this more demanding strategy. A similar argument may cause them not to switch to a higher gain and lower delay strategy at high track bandwidth when the track bandwidth gradually increases. In this case, they do not adopt the high gain strategy they exhibit when the track starts out with a high bandwidth. Again, it appears that subjects do not switch strategies to improve tracking performance to their demonstrated ability. In both cases, the 85 percentile measure indicates that performance is reduced during the period where they did not adopt the more effective strategy.

Rather than discussing the asymmetry in the subject's lead equalization in response to time varying plant dynamics, we want to draw attention to the slightly puzzling finding that a decreasing preview enabled subjects to track with a shorter delay time than when preview increases. It is as if subjects are better able to predict the reference track when they have been tracking it with preview for some time. We hypothesize that prolonged preview enables human operators to construct a more accurate mental model of the reference track characteristics which they then use in anticipating the track when preview drops below the critical value at which they, under static conditions, are able to equalize their inherent delay time.

The observed adaptation asymmetries indicate that tracking performance can not be predicted based on momentary task conditions alone. To establish a model of adaptation mechanisms and its driving forces, the recent history of changes in tracking conditions needs to be considered. Subjects appear to follow a lazy-controller's approach. Certain conditions cause them not to strive for "optimality". If the task becomes automatically easier, they do not always improve further. They also do not always adopt strategies that require more attention or result in a higher work load particularly when they have been tracking with a particular strategy from some time. Whether these asymmetric adaptation phenomena are the result of a subjectively established sufficient performance level or are the result of an asymmetry in inferring changes in not directly perceivable task conditions remains to be explored. One of the fundamental problems is our limited knowledge regarding a human operators internal performance criteria or cost function and how it is affected by recent history.

\section{Conclusion}

Our recursive system identifier [2] has demonstrated to be instrumental in analyzing a human operator's adaptive behavior. Its main strength lies in estimating time varying delay time which is a highly adaptable parameters in human operator models. It revealed asymmetries in human operator adaptation that may be of practical importance in applications where humans interact in slowly varying environ- ments. Unfortunately, space limitations forced us to touch on only a few of the observed asymmetries and leave a detailed discussion of these and the other data for a forthcoming journal paper. Topic of future research is to use this identifier in developing a model of human operator adaptation mechanisms during slowly varying tracking conditions. We believe that perceptual and cognitive aspects such as sensitivity to changes in control task parameters, perceived performance,perceived workload, mental or internal models and attention all play part in mediating adaptation.

\section{Acknowledgements}

This research was supported by NSF Grant number IRI9213822 and Nissan R\&D, Inc.

\section{References}

[1] E.R. Boer, Identification of Time Varying Systems with Applications to Adaptive Human Control. Ph.D. Dissertation, University of Illinois at Chicago, 1995.

[2] E.R. Boer and R.V. Kenyon, "Estimation of Time Varying Delay Time in Non-Stationary Linear Systems: An Approach to Monitor Human Operator Adaptation in Manual Tracking Tasks". IEEE Transactions on Systems, Man, and Cybernetics, Vol. 27A, No. 6, Nov. 1997 (In Press).

[3] J.I. Elkind and D.C. Miller, "Process of Adaptation by the Human Controller," Second Annual NASA-University Conference on Manual Control, NASA SP-128, pp. 47-63, 1966. [4] R.H. Hess, "A Model Based Theory for Analyzing Human Control Behavior," Advances in Man-Machine Systems Research, Vol. 2, JAI Press Inc., London, England, pp. 129175, 1985.

[5] H.R. Jex, J.D. McDonnell and A.V. Phatak, "A "Critical" Tracking Task for Manual Control Research," IEEE Transaction on Human Factors in Electronics, Vol. HFE-7, No. 4, pp. 138-145, Dec., 1966.

[6] A.V. Phatak and G.A. Bekey, "Model of the Adaptive Behavior of the Human Operator in Response to a Sudden Change in the Control Situation," IEEE Transactions on Man-Machine Systems, Vol. MMS-10, No. 3, pp. 72-80, Sep., 1969.

[7] T.B. Sheridan, Telerobotics, Automation, and Human Supervisory Control, The MIT Press, Cambridge, MA, 1992. [8] T.B. Sheridan and W.R. Ferrell, Man-Machine Systems, Information, Control, and Decision Models of Human Performance, The MIT press, Cambridge, MA, 1981.

[9] L.R. Young, "On Adaptive Manual Control," Ergonomics, Vol. 12, No. 4, pp. 635-675, July $1969^{3}$.

\footnotetext{
${ }^{3}$ Also appeared in IEEE Transactions on Man-Machine Systems, Vol. MMS-10, No. 4, pp. 292-331, Dec., 1969.
} 